\title{
COMPLEX GEODESICS AND VARIATIONAL CALCULUS FOR UNIVALENT FUNCTIONS
}

\author{
SAMUEL L. KRUSHKAL
}

\begin{abstract}
It turns out that complex geodesics in Teichmüller spaces with respect to their invariant metrics are intrinsically connected with variational calculus for univalent functions.

We describe this connection and show how geometric features associated to these metrics and geodesics provide deep distortion results for various classes of functions with quasiconformal extensions and create new phenomena which do not appear in the classical geometric function theory.
\end{abstract}

2010 Mathematics Subject Classification: Primary: 30C55, 30C62, 30C75, 30F60, 32F45; Secondary: 30F45, 46G20

Key words and phrases: Univalent, quasiconformal, Teichmüller space, infinite dimensional holomorphy, invariant metrics, complex geodesic, Grunsky inequalities, variational problem, functional

This paper is an extended version of my talk at the conference "Complex Analysis and Dynamical Systems VII". Our main goal is to reveal the intrinsic connection between the complex geodesics in Teichmüller spaces and extremals of the basic functionals in geometric function theory. This connection causes new phenomena that do not arise in the classical theory and provides an alternative approach to variational problems as well.

\section{Key theOREMS ON INVARIANT METRICS AND GEODESICS ON TEICHMÜLlER SPACES}

1.1. Teichmüller spaces. We shall use the notations $\widehat{\mathbb{C}}=\mathbb{C} \cup\{\infty\}$,

$$
\mathbb{D}=\{|z|<1\}, \mathbb{D}^{*}=\{z \in \widehat{\mathbb{C}}:|z|>1\}
$$

and consider two Teichmüller spaces: the universal Teichmüller space $\mathbf{T}=\mathbf{T}(\mathbb{D})$ and the Teichmüller space $\mathbf{T}_{1}=\mathbf{T}\left(\mathbb{D}^{0}\right)$ of the punctured disk $\mathbb{D}^{0}=\mathbb{D} \backslash\{0\}$.

Every Teichmüller space $\widetilde{\mathbf{T}}$ is a complex Banach manifold, thus it possesses the invariant Carathéodory and Kobayashi distances (the smallest and the largest among all holomorphically non-expanding metrics).

Denote these metrics by $c_{\widetilde{\mathbf{T}}}$ and $d_{\widetilde{\mathbf{T}}}$, and let $\tau_{\widetilde{\mathbf{T}}}$ be the intrinsic Teichmüller metric of this space canonically determined by quasiconformal maps. Their infinitesimal Finsler forms are defined on the tangent bundle $\mathcal{T} \widetilde{\mathbf{T}}$ of $\widetilde{\mathbf{T}})$, and satisfy

$$
c_{\widetilde{\mathbf{T}}}(\cdot, \cdot) \leq d_{\widetilde{\mathbf{T}}}(\cdot, \cdot) \leq \tau_{\widetilde{\mathbf{T}}}(\cdot, \cdot) .
$$

By the fundamental Royden-Gardiner theorem, the metrics $d_{\widetilde{\mathbf{T}}}$ and $\tau_{\widetilde{\mathbf{T}}}$ (and their infinitesimal forms) are equal; see, e.g. [6], [7, [8], [33].

Date: June 4, 2021 (geodvarCADS7rev.tex). 
So an important and still open question is how about the Carathéodory metric of $\widetilde{T}$ : does it also coincide with the Teichmüller metric? Our first theorem yields a positive answer for both spaces $\mathbf{T}$ and $\mathbf{T}_{1}$. Let $\widetilde{T}$ denote either from these spaces.

Theorem 1.1. The Carathéodory metric of the space $\widetilde{\mathbf{T}}$ coincides with its Kobayashi metric, hence all invariant non-expanding metrics on $\widetilde{\mathbf{T}}$ are equal its Teichmüller metric, and

$$
c_{\widetilde{T}}\left(X_{1}, X_{2}\right)=d_{\widetilde{T}}\left(X_{1}, X_{2}\right)=\tau_{\widetilde{T}}\left(X_{1}, X_{2}\right)=\inf \left\{d_{\mathbb{D}}\left(h^{-1}(\varphi), h^{-1}(\psi)\right): h \in \operatorname{Hol}(\mathbb{D}, \widetilde{\mathbf{T}})\right\},
$$

where $d_{\mathbb{D}}$ denotes the hyperbolic metric of the unit disk of curvature -4 .

Similarly, the infinitesimal forms of these metrics coincide with the Finsler metric $F_{\widetilde{\mathbf{T}}}(\varphi, v)$ generating $\tau_{\widetilde{\mathbf{T}}}$ and have holomorphic sectional curvature -4 .

This was known only for the universal Teichmüller space and underlies various applications. A new proof for this space is given in [22]. Its arguments (based on plurisubharmonic features and geometry of the Grunsky operator on univalent functions) can be extended to the second space $\mathbf{T}_{1}$. Since Theorem 1.1 underlies all other results of this paper, we present here its proof in the lines of 22] focusing mainly on the new case $\widetilde{\mathbf{T}}=\mathbf{T}_{1}$.

Recall that the universal Teichmüller space $\mathbf{T}=\mathbf{T}(\mathbb{D})$ is the space of quasisymmetric homeomorphisms of the unit circle $S^{1}=\partial \mathbb{D}$ quotiented by Möbius maps. The canonical complex Banach structure on $\mathbf{T}$ is defined by the quotient of the ball of Beltrami coefficients

$$
\operatorname{Belt}(\mathbb{D})_{1}=\left\{\mu \in L_{\infty}(\mathbb{C}): \mu \mid \mathbb{D}^{*}=0,\|\mu\|<1\right\},
$$

letting $\mu, \nu \in \operatorname{Belt}(\mathbb{D})_{1}$ be equivalent if the corresponding quasiconformal maps $f^{\mu}, f^{\nu} \in \Sigma^{0}$ coincide on $S^{1}$ and passing to Schwarzian derivatives

$$
S_{w}(z)=\left(\frac{w^{\prime \prime}(z)}{w^{\prime}(z)}\right)^{\prime}-\frac{1}{2}\left(\frac{w^{\prime \prime}(z)}{w^{\prime}(z)}\right)^{2} \quad\left(z \in \mathbb{D}^{*}, \quad w=f^{\mu} \mid \mathbb{D}^{*}\right) .
$$

Here $\Sigma^{0}$ denotes the collection of univalent functions

$$
f(z)=z+b_{0}+b_{1} z^{-1}+\ldots
$$

in $\mathbb{D}^{*}$ admitting quasiconformal extensions to the unit disk $\mathbb{D}$ with $f(1)=1 ; \quad f^{\mu}$ denotes the solution of the Beltrami equation $\bar{\partial} w=\mu \partial f$ on $\mathbb{C}$ with $\mu \in \operatorname{Belt}(\mathbb{D})_{1}$ and above normalization.

The Schwarzians $S_{f^{\mu}}$ run over a bounded domain in the Banach space B of hyperbolically bounded holomorphic functions on $\mathbb{D}^{*}$ with norm

$$
\|\varphi\|=\sup _{\mathbb{D}^{*}}\left(|z|^{2}-1\right)^{2}|\varphi(z)| .
$$

This domain models the space $\mathbf{T}$, and the defining projection $\phi_{\mathbf{T}}: \mu \rightarrow S_{f^{\mu}}$ is holomorphic as a $\operatorname{map} L_{\infty}(\mathbb{D}) \rightarrow \mathbf{B}$.

The intrinsic Teichmüller metric of the space $\mathbf{T}$ is given by

$$
\tau_{\mathbf{T}}\left(\phi_{\mathbf{T}}(\mu), \phi_{\mathbf{T}}(\nu)\right)=\frac{1}{2} \inf \left\{\log K\left(w^{\mu_{*}} \circ\left(w^{\nu_{*}}\right)^{-1}\right): \mu_{*} \in \phi_{\mathbf{T}}(\mu), \nu_{*} \in \phi_{\mathbf{T}}(\nu)\right\},
$$

where $\phi_{\mathbf{T}}$ is the factorizing holomorphic projection $\operatorname{Belt}(\mathbb{D})_{1} \rightarrow \mathbf{T}$ and

$$
K\left(w^{\mu}\right)=\left(1+\|\mu\|_{\infty}\right) /\left(1-\|\mu\|_{\infty}\right)
$$

denotes the maximal quasiconformal dilatation of the map $w^{\mu}$. This metric is the integral form of the infinitesimal Finsler metric

$$
F_{\mathbf{T}}\left(\phi_{\mathbf{T}}(\mu), \phi_{\mathbf{T}}^{\prime}(\mu) \nu\right)=\inf \left\{\left\|\nu_{*} /\left(1-|\mu|^{2}\right)\right\|_{\infty}: \phi_{\mathbf{T}}^{\prime}(\mu) \nu_{*}=\phi_{\mathbf{T}}^{\prime}(\mu) \nu\right\}
$$

on the tangent bundle $\mathcal{T} \mathbf{T}$ of $\mathbf{T}$. 
The space $\mathbf{T}_{1}=\mathbf{T}\left(\mathbb{D}^{0}\right)$ is canonically isomorphic to the subspace $\mathbf{T}(\Gamma)=\mathbf{T} \cap \mathbf{B}(\Gamma)$, where $\Gamma$ is a cyclic parabolic Fuchsian group in $\mathbb{D}$ uniformizing the punctured disk $\mathbb{D}^{0}$ and

$$
\mathbf{B}(\Gamma)=\left\{\varphi \in \mathbf{B}:(\varphi \circ \gamma)\left(\gamma^{\prime}\right)^{2}=\varphi, \gamma \in \Gamma\right\} \quad \text { in } \mathbb{D}^{*} .
$$

By [1], this space is biholomorphically equivalent to the Bers fiber space $\mathbf{F}(\mathbf{T})$ over universal Teichmüller space, which underlies the proof of Theorem 1.1 for $\mathbf{T}_{1}$.

1.2. Complex geodesics. If $X$ is a domain in a complex Banach space $E$ endowed with a pseudodistance $\rho_{X}$, then a holomorphic map $h: \mathbb{D} \rightarrow X$ is called a complex $\rho$-geodesic if there exist $t_{1} \neq t_{2}$ in $\mathbb{D}$ such that

$$
d_{\mathbb{D}}\left(t_{1}, t_{2}\right)=\rho_{X}\left(h\left(t_{1}\right), h\left(t_{2}\right)\right)
$$

one says also that the points $h\left(t_{1}\right)$ and $h\left(t_{2}\right)$ can be joined by a complex $\rho$-geodesic (see [40]).

If $h$ is a complex $c_{X}$-geodesic then it also is $d_{X}$-geodesic and the above equality holds for all points $t_{1}, t_{2} \in \mathbb{D}$, so $h(\mathbb{D})$ is a holomorphic disk in $X$ hyperbolically isometric to $\mathbb{D}$.

The second basic theorem follows from Theorem 1.1 and the properties of extremal quasiconformal maps.

Theorem 1.2. (i) Any two points of the space $\widetilde{\mathbf{T}}$ can be joined by a complex geodesic. The geodesic joining a Strebel point with the base point is unique and defines the corresponding Teichmüller extremal disk.

(ii) For any point $\varphi \in \widetilde{\mathbf{T}}$ and any nonzero tangent vector $v$ at this point, there exists at least one complex geodesic $h: \mathbb{D} \rightarrow \widetilde{\mathbf{T}}$ such that $h(0)=\varphi$ and $h^{\prime}(0)$ is collinear to $v$.

1.3. The coincidence of invariant metrics and existence of complex geodesics was known only for convex domains in dual Banach spaces (see [3]). Duality is needed to apply the Alaoglu-Banach theorem.

Theorem 1.1 implies, together with the definition of complex geodesics, that these geodesics in $\widetilde{\mathbf{T}}$ are the Teichmüller geodesic disks in this space.

Such disks are uniquely determined for Strebel points; on the other hand, Tanigawa constructed in [38] the extremal Beltrami coefficients $\mu_{0}$ with nonconstant $\left|\mu_{0}(z)\right|<\left\|\mu_{0}\right\|_{\infty}$ on a set of positive measure for which there exist infinitely many distinct geodesic segments in the universal Teichmüller space $\mathbf{T}$ joining the points $\phi_{\mathbf{T}}(\mathbf{0})$ and $\phi_{\mathbf{T}}\left(\mu_{0}\right)$. All these segments belong to different complex geodesics joining the indicated points.

\section{Proof of Theorem 1.1}

The proof of this underlying theorem involves essentially the properties of the Grunsky coefficients of univalent functions. As an important consequence of this theorem, one obtain Theorem 1.2 on complex geodesics of these metrics which will be applied to variational calculus.

$\mathbf{1}^{\mathbf{0}}$. Auxiliary lemmas. Consider the space $\Sigma^{0}$ of the univalent $\widehat{\mathbb{C}}$-holomorphic functions $f(z)=$ $z+b_{0}+b_{1} z^{-1}+\ldots$ on the disk $\mathbb{D}^{*}$ with quasiconformal extension to $\widehat{\mathbb{C}}$ satisfying $f(1)=1$. Their Grunsky coefficients $\alpha_{m n}$ are defined via

$$
\log \frac{f(z)-f(\zeta)}{z-\zeta}=-\sum_{m, n=1}^{\infty} \alpha_{m n} z^{-m} \zeta^{-n}, \quad(z, \zeta) \in\left(\mathbb{D}^{*}\right)^{2}
$$


choosing the branch of the logarithmic function which vanishes as $z=\zeta \rightarrow \infty$. The quantity

$$
\varkappa(f):=\sup \left\{\left|\sum_{m, n=1}^{\infty} \sqrt{m n} \alpha_{m n} x_{m} x_{n}\right|\right\}
$$

where the supremum is taken over the points of the unit sphere $S\left(l^{2}\right)=\{\|\mathbf{x}\|=1\}$ in the Hilbert space of sequences $\mathbf{x}=\left(x_{n}\right)$ with $\|\mathbf{x}\|^{2}=\sum_{1}^{\infty}\left|x_{n}\right|^{2}$, is called the Grunsky norm of $f$, and by a result of Grunsky [11] the inequality $\varkappa(f) \leq 1$ is the necessary and sufficient condition for univalence of $f$ in the disk $\mathbb{D}^{*}$.

The coefficients $\alpha_{m n}$ are polynomials of the initial Taylor coefficients $b_{1}, b_{2}, \ldots, b_{m+n_{1}}, \ldots$ of $f$, hence depend holomorphically on Beltrami differentials $\mu \in \operatorname{Belt}(\mathbb{D})_{1}$ and on the Schwarzian derivatives $S_{f} \in \mathbf{B}$ of these functions (see, e.g., [8], [15]). This generates for a fixed $\mathbf{x}=\left(x_{n}\right) \in S\left(l^{2}\right)$ the holomorphic map

$$
h_{\mathbf{x}}\left(S_{f}\right)=\sum_{m, n=1}^{\infty} \sqrt{m n} \alpha_{m n}\left(S_{f}\right) x_{m} x_{n}: \mathbf{T} \rightarrow \mathbb{D},
$$

and $\sup _{\mathbf{x} \in S\left(l^{2}\right)}\left|h_{\mathbf{x}}\left(S_{f}\right)\right|=\varkappa(f)$. Both the Teichmüller and Grunsky norms of $f$ are continuous plurisubharmonic functions of $S_{f}$ on $\mathbf{T}$ (see, e.g. [18], 21]).

Note that the convergence and holomorphy of the series (2.1) simply follow from the inequalities

$$
\left|\sum_{m=j}^{M} \sum_{n=l}^{N} \sqrt{m n} \alpha_{m n} x_{m} x_{n}\right|^{2} \leq \sum_{m=j}^{M}\left|x_{m}\right|^{2} \sum_{n=l}^{N}\left|x_{n}\right|^{2}
$$

(for any finite $M, N$ ) which, in turn, are a consequence of the classical area theorem (see, e.g., [30, p. 61]).

Let $A_{1}(\mathbb{D})$ be the subspace of $L_{1}(\mathbb{D})$ formed by holomorphic functions in $\mathbb{D}$, and

$$
A_{1}^{2}=\left\{\psi \in A_{1}(\mathbb{D}): \psi=\omega^{2}\right\} ;
$$

this set consists of the integrable holomorphic functions on $\mathbb{D}$ having only zeros of even order. In fact, all $\psi \in A_{1}^{2}$ are of the form

$$
\psi(z)=\frac{1}{\pi} \sum_{m+n=2}^{\infty} \sqrt{m n} x_{m} x_{n} z^{m+n-2},
$$

with $\|\mathbf{x}\|_{l^{2}}=\|\omega\|_{L_{2}}$. Put

$$
<\mu, \psi>\mathbb{D}=\iint_{\mathbb{D}} \mu(z) \psi(z) d x d y, \quad \mu \in L_{\infty}(\mathbb{D}), \psi \in L_{1}(\mathbb{D}) \quad(z=x+i y)
$$

and

$$
\alpha_{\mathbb{D}}(f)=\sup \left\{\left|\left\langle\mu_{0}, \psi\right\rangle_{\mathbb{D}}\right|: \psi \in A_{1}^{2},\|\psi\|_{A_{1}(\mathbb{D})}=1\right\} .
$$

The Grunsky norm of every $f \in \Sigma^{0}$ is dominated by its Teichmüller norm, i.e., $\varkappa(f) \leq k(f)[25$. We shall need a stronger estimate given by

Lemma 2.1. [16], 21] For all $f \in \Sigma^{0}$,

$$
\varkappa(f) \leq k \frac{k+\alpha_{\mathbb{D}}(f)}{1+\alpha_{\mathbb{D}}(f) k}, \quad k=k(f),
$$

and $\varkappa(f)<k$ unless

$$
\alpha_{\mathbb{D}}(f)=\left\|\mu_{0}\right\|_{\infty}
$$

and the last equality is equivalent to $\varkappa(f)=k(f)$. Moreover, for small $\|\mu\|_{\infty}$,

$$
\varkappa_{r}(f)=\sup \left|\langle\mu, \psi\rangle_{\mathbb{D}}\right|+O\left(\|\mu\|_{\infty}^{2}\right), \quad\|\mu\|_{\infty} \rightarrow 0,
$$


with the same supremum as in (2.3).

If $\varkappa(f)=k(f)$ and the equivalence class of $f$ (the collection of maps equal $f$ on $S^{1}=\partial \mathbb{D}^{*}$ ) is a Strebel point, then $\mu_{0}$ is necessarily of the form

$$
\mu_{0}=\left\|\mu_{0}\right\|_{\infty}\left|\psi_{0}\right| / \psi_{0} \text { with } \psi_{0} \in A_{1}^{2} .
$$

Geometrically, the equality (2.3) means the equality of the Carathéodory and Teichmüller distances on the geodesic disk

$$
\mathbb{D}\left(\mu_{0}\right)=\left\{\phi_{\mathbf{T}}\left(t \mu_{0} /\left\|\mu_{0}\right\|\right): t \in \mathbb{D}\right\} \subset \mathbf{T}
$$

(compare with Kra's theorem for finite dimensional Teichmüller spaces [14]).

For functions $f \in \Sigma^{0}$ holomorphic in the closed disk $\overline{\mathbb{D}^{*}}$, the relation (2.4) was also obtained (by a different method) in [27].

One defines for $f \in \Sigma^{0}$ the complex homotopy

$$
f_{t}(z)=t f(z / t)=z+b_{0} t+b_{1} t^{2} z^{-1}+b_{2} t^{3} z^{-2}+\ldots: \mathbb{D}^{*} \times \mathbb{D} \rightarrow \widehat{\mathbb{C}} .
$$

Then $S_{f_{t}}(z)=t^{-2} S_{f}\left(t^{-1} z\right)$, and moreover, this point-wise map determines a holomorphic map

$$
\beta_{f}(t)=S_{f_{t}}(\cdot): \mathbb{D} \rightarrow \mathbf{B} .
$$

The homotopy disks $\mathbb{D}\left(S_{f}\right)=\beta_{f}(\mathbb{D})$ have only cuspidal critical points (are branched simply connected Riemann surfaces placed in $\mathbf{T}$ ) and foliate the space $\mathbf{T}$.

Each homotopy map $f_{t}$ admits an extremal extension to $\mathbb{D}$ of Teichmüller type with dilatation $k\left(f_{t}\right) \leq k(f)|t|^{2}$. This bound is sharp and is improved for

$$
f(z)=z+b_{0}+b_{m} z^{-m}+\ldots \quad\left(m>1, b_{m} \neq 0\right),
$$

via $k\left(f_{t}\right) \leq k(f)|t|^{m+1}$. Due to [24],

$$
k\left(f_{t}\right)=\frac{m+1}{2}\left|b_{m}\right||t|^{m+1}+O\left(t^{m+2}\right), \quad t \rightarrow 0 .
$$

The following lemma belongs to Kühnau [27, see also [24, Section 6].

Lemma 2.2. In the case $b_{1} \neq 0$, there holds for sufficiently small $|t| \leq r_{0}(f)$ the equality $\varkappa\left(f_{t}\right)=$ $k\left(f_{t}\right)$.

Indeed, due to [27, for small $|t|$ the extremal quasiconformal extension of $f_{t}$ to $D$ is defined by a nonvanishing holomorphic quadratic differential. Generically, $r_{0}(f)<1$, which is connected with critical points of the homotopy disk.

Note that $S_{f}(z)=-6 b_{1} z^{-4}+O\left(z^{-5}\right)$, so $b_{1}=0$ only for $f$ with

$$
\lim _{z \rightarrow \infty} z^{4} S_{f}(z)=-6 b_{1}=0 .
$$

It suffices to prove the theorem for the set of $S_{f} \in \mathbf{T}$ such that $b_{1} \neq 0$ which is open and dense in T.

The following lemma is a special case of a local existence theorem from [15].

Lemma 2.3. Let $D$ be a simply connected domain on the Riemann sphere $\widehat{\mathbb{C}}$. Assume that there are a set $E$ of positive two-dimensional Lebesgue measure and a finite number of points $z_{1}, z_{2}, \ldots, z_{m}$ distinguished in $D$. Let $\alpha_{1}, \alpha_{2}, \ldots, \alpha_{m}$ be non-negative integers assigned to $z_{1}, z_{2}, \ldots, z_{m}$, respectively, so that $\alpha_{j}=0$ if $z_{j} \in E$.

Then, for a sufficiently small $\varepsilon_{0}>0$ and $\varepsilon \in\left(0, \varepsilon_{0}\right)$, and for any given collection of numbers $w_{s j}, s=0,1, \ldots, \alpha_{j}, j=1,2, \ldots, m$ which satisfy the conditions $w_{0 j} \in D$,

$$
\left|w_{0 j}-z_{j}\right| \leq \varepsilon, \quad\left|w_{1 j}-1\right| \leq \varepsilon, \quad\left|w_{s j}\right| \leq \varepsilon\left(s=0,1, \ldots a_{j}, j=1, \ldots, m\right),
$$


there exists a quasiconformal self-map $h$ of $D$ which is conformal on $D \backslash E$ and satisfies

$$
h^{(s)}\left(z_{j}\right)=w_{s j} \quad \text { for all } s=0,1, \ldots, \alpha_{j}, j=1, \ldots, m .
$$

Moreover, the Beltrami coefficient $\mu_{h}(z)=\partial_{\bar{z}} h / \partial_{z} h$ of $h$ on $E$ satisfies $\left\|\mu_{h}\right\|_{\infty} \leq M \varepsilon$. The constants $\varepsilon_{0}$ and $M$ depend only upon the sets $D, E$ and the vectors $\left(z_{1}, \ldots, z_{m}\right)$ and $\left(\alpha_{1}, \ldots, \alpha_{m}\right)$.

If the boundary $\partial D$ is Jordan or is $C^{l+\alpha}$-smooth, where $0<\alpha<1$ and $l \geq 1$, we can also take $z_{j} \in \partial D$ with $\alpha_{j}=0$ or $\alpha_{j} \leq l$, respectively.

The following lemma relies on classical results on compactness in the dual week* topology and is a consequence of the Alaoglu-Bourbaki theorem.

Lemma 2.4. Let $Y$ be reflexive and let $\Omega$ be a bounded set in $\operatorname{Hol}(G, Y)$. Then any sequence from $\Omega$ contains a subsequence which weakly converges to a holomorphic map from $G$ to $Y$.

Hence, the compactness (strong or weak) is actually required only for the image of $G$ in $Y$ under a given family of maps.

In our case $Y=\mathbb{C}$, and for each $x \in \widetilde{\mathbf{T}}$ its orbit $h(x)$ is located in the unit disk which is compact. This implies that for any point $x_{0}$ from $\widetilde{\mathbf{T}}$ there exists a holomorphic map $h_{0}: \widetilde{\mathbf{T}} \rightarrow \mathbb{D}$ with $h_{0}(0)=0$ and $d_{\mathbb{D}}\left(0, h_{0}\left(x_{0}\right)\right)=c_{\mathbf{T}}\left(\mathbf{0}, x_{0}\right)$.

$\mathbf{2}^{\mathbf{0}}$. Case $\widetilde{\mathbf{T}}=\mathbf{T}$. Consider for the Schwarzians $S_{f} \in \mathbf{T}$ their homotopy disks $\Delta\left(S_{f}\right)$. It follows from Lemmas 2.1 and 2.2 that for small $|t| \leq r_{0}(f)$,

$$
c_{\mathbf{T}}\left(\mathbf{0}, S_{f_{t}}\right)=d_{\mathbf{T}}\left(\mathbf{0}, S_{f_{t}}\right)=\tau_{\mathbf{T}}\left(\mathbf{0}, S_{f_{t}}\right)=\tanh ^{-1}|t|,
$$

and similarly for the corresponding infinitesimal metrics. Our goal now is to extend this equality to all points of $\Delta\left(S_{f}\right)$.

We first illustrate the arguments on the universal Teichmüller space $\mathbf{T}$. The proof for the space $\mathbf{T}_{1}=\mathbf{T}\left(\mathbb{D}^{0}\right)$ will be given separately in the next step.

Consider in the tangent bundle $\mathcal{T}(\mathbf{T})=\mathbf{T} \times \mathbf{B}$ the holomorphic disks $\widehat{\mathbb{D}}\left(S_{f}\right)$ over the homotopy disk of $f$ formed by the points $\left(\varphi_{t}, v\right)$, where $v=\phi_{\mathbf{T}}^{\prime}\left[\varphi_{t}\right] \mu \in \mathbf{B}$ is a tangent vector to $\mathbf{T}$ at the point $\varphi_{t}=S_{f_{t}}$, and $\mu$ runs over the ball

$$
\operatorname{Belt}\left(D_{\varphi_{t}}\right)_{1}=\left\{\mu \in L_{\infty}(\mathbb{C}): \mu \mid D_{\varphi_{t}}^{*}=0,\|\mu\|_{\infty}<1\right\}
$$

Here $D_{\varphi}$ and $D_{\varphi}^{*}$ denote the images of $\mathbb{D}$ and $\mathbb{D}^{*}$ under $f=f_{\varphi} \in \Sigma^{0}$ with $S_{f}=\varphi$.

Pick the unit tangent vectors $v$ to $\mathbf{T}$ at the origin and holomorphic maps $\widehat{h}_{v}: \quad \mathbf{T} \rightarrow \mathbb{D}$ with $\widehat{h}_{v}(\mathbf{0})=0$ and maximal lengths $\left|\widehat{d h}_{v}(\mathbf{0}) v\right|=\mathcal{C}_{\mathbf{T}}(\mathbf{0}, v)$ (given by Lemma 2.4) and apply these maps to pulling back the hyperbolic metric on the unit disk

$$
d s=|d t| /\left(1-|t|^{2}\right)
$$

of Gaussian curvature -4 onto the disks $\widehat{\mathbb{D}}\left(S_{f}\right)$. Then we obtain on these disks the logarithmically subharmonic metrics $d s=\widehat{\lambda}_{\widehat{h}_{v}}(t)|d t|$ with

$$
\widehat{\lambda}_{\widehat{h}_{v}}(t)=\widehat{h}_{v}^{*} \lambda_{\mathbb{D}}=\frac{\left|\widehat{h}_{v}^{\prime}(t)\right|}{1-\left|\widehat{h}_{v}(t)\right|^{2}},
$$

having Gaussian curvature -4 at noncritical points. The upper envelope of these metrics

$$
\lambda_{0}(t)=\sup _{\widehat{h}_{v}} \widehat{\lambda}_{\widehat{h}_{v}}(t),
$$

followed by its upper semicontinuous regularization depends only on the points $\varphi \in \mathbf{T}$, hence it descends to a logarithmically subharmonic metric $\lambda_{0}$ on the underlying disk $\mathbb{D}\left(S_{f}\right)$. The underlying 
metric $\lambda_{0}$ has at each of its noncritical point $t_{0}$ a supporting metric of curvature -4 , thus the generalized Gaussian curvature of $\lambda_{0}$ satisfies $\kappa_{\lambda_{0}} \leq-4$, or equivalently,

$$
\Delta \log \lambda_{0} \geq 4 \lambda_{0}^{2} .
$$

Here $\Delta$ means the generalized Laplacian

$$
\Delta \lambda(t)=4 \liminf _{r \rightarrow 0} \frac{1}{r^{2}}\left\{\frac{1}{2 \pi} \int_{0}^{2 \pi} \lambda\left(t+r e^{i \theta}\right) d \theta-\lambda(t)\right\} \quad(0 \leq \lambda(t)<\infty) .
$$

Now the metrics $\lambda_{\mathcal{C}}$ and $\lambda_{\mathcal{K}}$ can be compared on the disk $\mathbb{D}\left(S_{f}\right)$ by Minda's maximum principle for solutions of (2.8) given by

Lemma 2.5. 29] If a function $u: \Omega \rightarrow[-\infty,+\infty)$ is upper semicontinuous in a domain $\Omega \subset \mathbb{C}$ and its generalized Laplacian satisfies the inequality $\Delta u(z) \geq K u(z)$ with some positive constant $K$ at any point $z \in \Omega$, where $u(z)>-\infty$, and if $\lim \sup u(z) \leq 0$ for all $\zeta \in \partial \Omega$, then either $u(z)<0$ for all $z \in \Omega$ or else $u(z)=0$ for all $z \in \Omega$.

It is applied to the ratio $u=\log \left(\lambda_{\mathcal{C}} / \lambda_{\mathcal{K}}\right)=\log \lambda_{\mathcal{C}}-\log \lambda_{\mathcal{K}}$. One needs to consider only the points $S_{f_{t}}$ with $|t|>r_{0}$. In view of the upper semicontinuity of both metrics the set $U_{0}$ of points $t \in \mathbb{D},|t|>r_{0}$, where $\lambda_{\mathcal{C}}(t)<\lambda_{\mathcal{K}}(t)$, is open. Arguing similar to [29], one derives from (2.6) and Lemma 2.5 that $\lambda_{\mathcal{C}}=\lambda_{\mathcal{K}}$ on $U_{0}$. Continuing in a similar way, one extends this equality first to all noncritical points of the disk $\mathbb{D}\left(S_{f}\right)$ and then by continuity of metrics to the whole homotopy disk. This also provides the equality of the global distances $c_{\mathbf{T}}$ and $d_{\mathbf{T}}$ on this disk.

Now, by applying the infinitesimal analog of Montel's normality theorem and Lemma 2.4, one gets, letting $t \rightarrow 1$,

$$
c_{\mathbf{T}}\left(\mathbf{0}, S_{f}\right)=d_{\mathbf{T}}\left(\mathbf{0}, S_{f}\right) .
$$

The case of two arbitrary points $\varphi_{1}, \varphi_{2} \in T$ is reduced to (2.9) in a standard way using the right translations of $\operatorname{Belt}(\mathbb{D})_{1}$. The details will be given in the next step.

Remark. We have distinguished in the above proof the canonical disk $\mathbb{D}^{*}$ as the base point of $\mathbf{T}$. In fact, the arguments work for any other base point (quasidisk $D^{*}$ ) of this space. Accordingly, one can define by (2.1) the generalized Grunsky coefficients $\alpha_{m n}(f)$ for univalent functions $f(z)$ in this quasidisk $D^{*}$ and construct similar to $(2.2)$ the holomorphic maps $h_{\mathbf{x}}\left(S_{f}\right): \mathbf{T} \rightarrow \mathbb{D}$ moving the base point $D^{*}$ into the origin (see [22]).

$\mathbf{3}^{\mathbf{0}}$. Proof for the space $\mathbf{T}_{1}$. First recall that the elements of the space $\mathbf{T}_{1}=\mathbf{T}(\mathbb{D} \backslash\{0\})$ are the equivalence classes of Beltrami coefficients $\mu \in \operatorname{Belt}(\mathbb{D})_{1}$ so that the corresponding quasiconformal automorphisms $w^{\mu}$ of the unit disk coincide on both boundary components (unit circle $S^{1}$ and the puncture $z=0$ ) and are homotopic on $\mathbb{D} \backslash\{0\}$. This space can be endowed with a canonical complex structure of a complex Banach manifold and embedded into $\mathbf{T}$ using uniformization.

Namely, the punctured disk $\mathbb{D} \backslash\{0\})$ is conformally equivalent to the factor $\mathbb{D} / \Gamma$, where $\Gamma$ is a cyclic parabolic Fuchsian group acting discontinuously on $\mathbb{D}$ and $\mathbb{D}^{*}$. The functions $\mu \in L_{\infty}(\mathbb{D})$ are lifted to $\mathbb{D}$ as the Beltrami $(-1,1)$-measurable forms $\widetilde{\mu} d \bar{z} / d z$ in $\mathbb{D}$ with respect to $\Gamma$, i.e., via $(\widetilde{\mu} \circ \gamma) \overline{\gamma^{\prime}} / \gamma^{\prime}=\widetilde{\mu}, \gamma \in \Gamma$, forming the Banach space $L_{\infty}(\mathbb{D}, \Gamma)$.

We extend these $\widetilde{\mu}$ by zero to $\mathbb{D}^{*}$ and consider the unit ball Belt $(\mathbb{D}, \Gamma)_{1}$ of $L_{\infty}(\mathbb{D}, \Gamma)$. Then the corresponding Schwarzians $S_{w^{\tilde{\mu}} \mid \mathbb{D}^{*}}$ belong to $\mathbf{T}$. Moreover, $\mathbf{T}_{1}$ is canonically isomorphic to the subspace $\mathbf{T}(\Gamma)=\mathbf{T} \cap \mathbf{B}(\Gamma)$, where $\mathbf{B}(\Gamma)$ consists of elements $\varphi \in \mathbf{B}$ satisfying $(\varphi \circ \gamma)\left(\gamma^{\prime}\right)^{2}=\varphi$ in $\mathbb{D}^{*}$ for all $\gamma \in \Gamma$.

Due to the Bers isomorphism theorem, the space $\mathbf{T}_{1}$ is biholomorphically equivalent to the Bers fiber space

$$
\left.\mathcal{F}(\mathbf{T})=\left\{\phi_{\mathbf{T}}(\mu), z\right) \in \mathbf{T} \times \mathbb{C}: \mu \in \operatorname{Belt}(\mathbb{D})_{1}, z \in w^{\mu}(\mathbb{D})\right\}
$$


over the universal Teichmüller space with holomorphic projection $\pi(\psi, z)=\psi$ (see [1]). This fiber space is a bounded domain in $\mathbf{B} \times \mathbb{C}$.

Note that now the admissible quadratic differentials defining Teichmüller extremal coefficients $\mu_{0} \in \operatorname{Belt}(\mathbb{D})_{1}$ must be integrable and holomorphic only on $\mathbb{D} \backslash\{0\}$, thus can have simple pole at $z=0$, i.e., $\mu_{0}=k\left|\psi_{0}\right| / \psi_{0}$ with

$$
\psi_{0}(z)=c_{-1} z^{-1}+c_{0}+c_{1} z+\ldots, \quad 0<|z|<1 .
$$

To prove the theorem for the space $\mathbf{T}_{1}$, we establish the equality of invariant metrics on this fiber space. We again model the space $\mathbf{T}$ as a domain in the space $\mathbf{B}$ formed by the Schwarzians $S_{f^{\mu}}$ of functions $f^{\mu} \in \Sigma^{0}$.

We associate with $f^{\mu}$ the odd function

$$
\mathcal{R} f^{\mu}(z):=\left(f^{\mu}\left(z^{2}\right)-f^{\mu}(0)\right)^{1 / 2}=z+\frac{b_{0}-f^{\mu}(0)}{2 z}+\frac{b_{3}^{\prime}}{z^{3}}+\ldots
$$

whose Grunsky coefficients $\alpha_{m n}\left(\mathcal{R} f^{\mu}\right)$ are represented as polynomials of the first Taylor coefficients of the original function $f^{\mu}$ and of $a=f^{\mu}(0)$. Hence, $\alpha_{m n}\left(\mathcal{R} f^{\mu}\right)$ depend holomorphically on the Schwarzians $\varphi=S_{f^{\mu}} \in \mathbf{T}$ and on values $f^{\mu}(0)$, i.e., on pairs $X=(\varphi, a)$ which are the points of the fiber space $\mathcal{F}(\mathbf{T})$. This joint holomorphy follows from Hartog's theorem on separately holomorphic functions extended to Banach domains.

The square root transform act on quadratic differentials $\psi_{0} d z^{2}$ via

$$
\mathcal{R}_{*} \psi_{0}=\psi_{0}\left(z^{2}\right) 4 z^{2} d z^{2}=\left(4 c_{-1}+\widetilde{c}_{2} z^{2}+\ldots\right) d z^{2} ;
$$

so one can apply to $\mathcal{R} f^{\mu}$ Lemmma 2.2 .

We construct by $(2.2)$ for $\mathcal{R} f^{\mu}$ the corresponding holomorphic functions

$$
\widehat{h}_{\mathbf{x}}\left(S_{f}\right)=\sum_{m, n=1}^{\infty} \sqrt{m n} \alpha_{m n}\left(S_{\mathcal{R} f}\right) x_{m} x_{n}: \mathbf{T} \rightarrow \mathbb{D}
$$

mapping the domain $\mathcal{F}(\mathbf{T})$ to the unit disk and satisfying

$$
\sup _{\mathbf{x} \in S\left(l^{2}\right)} \widehat{h}_{\mathbf{x}}\left(S_{f}\right)=\varkappa(\mathcal{R} f) \text {. }
$$

Using Lemma 2.3, we can now restrict ourselves by $f \in \Sigma^{0}$ with

$$
f(0) \neq b_{0} .
$$

Indeed, there exists by this lemma a quasiconformal automorphisms $W_{\varepsilon}$ of the plane $\widehat{\mathbb{C}}$, which is conformal outside a domain $E$ located in a neighborhood of the infinity point, and satisfies

$$
W_{\varepsilon}(z)=z+a_{2, \varepsilon} z^{2}+a_{3, \varepsilon} z^{3}+\ldots \quad \text { for }|z| \leq r_{1}(\varepsilon) ; \quad a_{2, \varepsilon} \neq 0, \quad W_{\varepsilon}(1)=1, W_{\varepsilon}(\infty)=\infty .
$$

Then

$$
g_{\varepsilon}(z)=1 / W_{\varepsilon}(1 / z)=z-a_{2, \varepsilon}+b_{1, \varepsilon} z^{-1}+\ldots
$$

is conformal outside a small neighborhood of the origin, and for any $f \in \Sigma^{0}$ with $f(0)=b_{0}$ the compozed map $g_{\varepsilon} \circ f$ satisfies (2.12).

For $f$ satisfying $(2.12)$, we have by $(2.10) b_{1}(\mathcal{R} f) \neq 0$, and Lemma 2.2 yields

$$
\varkappa(\mathcal{R} f)=k(\mathcal{R} f)=k(f), \quad \text { for small }|t| \leq r_{*}(f) .
$$

Put $X_{f}=\left(S_{f}, f(0)\right)$ and consider in $\mathcal{F}(\mathbf{T})$ the homotopy disks

$$
\mathbb{D}\left(X_{f}\right)=\left\{X_{f_{t}}=\left(S_{f_{t}}, f(0) t\right):|t|<1\right\} .
$$

It follows from (2.11) and (2.13) that for all $|t| \leq r_{*}$,

$$
c_{\mathcal{F}(\mathbf{T})}\left(\mathbf{0}, X_{t}\right)=\tau_{\mathcal{F}(\mathbf{T})}\left(\mathbf{0}, X_{t}\right)=d_{\mathcal{F}(\mathbf{T})}\left(\mathbf{0}, X_{t}\right)=\tanh ^{-1}|t| .
$$


Now, arguing similar to the case $\mathbf{2}^{\mathbf{0}}$, one extends these equalities to all $|t|<1$ and then to the initial point $X=\left(S_{f}, f(0)\right.$.

Since the spaces $\mathbf{T}_{1}$ and $\mathcal{F}(\mathbf{T})$ are biholomorphically equivalent, the corresponding metrics on $\mathbf{T}_{1}$ obey similar relations, which provides that for any point $X \in \mathbf{T}_{1}$ its distance from the base point $X_{0}$ in any invariant (no-expanding) metric is equal to the Teichmüller distance

$$
c_{\mathbf{T}_{1}}\left(X_{0}, X\right)=\tau_{\mathbf{T}_{1}}\left(X_{0}, X\right)=d_{\mathbf{T}_{1}}\left(X_{0}, X\right) .
$$

It remains to establish the equality of distances between two arbitrary points $X_{1}, X_{2}$ in $\mathbf{T}_{1}$.

To reduce this case to (2.14), we uniformize the base point $X_{0}=\mathbb{D} \backslash\{0\}$ (with fixed homotopy class) of this space by a cyclic parabolic Fuchsian group $\Gamma_{0}$ acting on the unit disk (using the universal covering $\pi: \mathbb{D} \rightarrow X_{0}$ with $\pi(0)=0$ ) and embed the space $\mathbf{T}_{1}$ holomorphically into $\mathbf{T}$ via

$$
\mathbf{T}_{1}=\mathbf{T} \cap \mathbf{B}\left(\mathbb{D}^{*}, \Gamma_{0}\right)=\operatorname{Belt}\left(\mathbb{D}, \Gamma_{0}\right) / \sim
$$

(where the equivalence relation commutate with the homotopy of quasiconformal homeomorphisms of the surfaces). This preserves all invariant distances on $\mathbf{T}_{1}$.

Now, fix a Beltrami coefficient $\mu \in \operatorname{Belt}\left(\mathbb{D}, \Gamma_{0}\right)_{1}$ so that $X_{1}=w^{\nu}\left(X_{0}\right)$ as marked surfaces (i.e., with prescribed homotopy classes) and apply the change rule for Beltrami coefficients: for any $\mu, \nu \in \operatorname{Belt}(\mathbb{C})_{1}$, the solutions $w^{\mu}$ of the corresponding Beltrami equation $\partial_{\bar{z}} w=\mu \partial_{z} w$ on $\widehat{\mathbb{C}}$ satisfy $w^{\mu} \circ w^{\nu}=w^{\sigma_{\nu}(\mu)}$, with

where

$$
\sigma_{\nu}(\mu)=\left(\nu+\mu^{*}\right) /\left(1+\bar{\nu} \mu^{*}\right)
$$

$$
\mu^{*}(z)=\mu \circ w^{\nu}(z) \overline{\partial_{z} w^{\nu}(z)} / \partial_{z} w^{\nu}(z) .
$$

Thus, for $\nu$ fixed, $\sigma_{\nu}(\mu)$ depends holomorphically on $\mu$ as a map $L_{\infty}(\mathbb{C}) \rightarrow L_{\infty}(\mathbb{C})$, which defines a holomorphic automorphism $\sigma_{\mu}$ of the ball Belt $\left(\mathbb{D}, \Gamma_{0}\right)$ preserving its Teichmüller metric. This automorphism is compatible with holomorphic factorizing projections $\phi_{\mathbf{T}_{1}}$ and $\phi_{\mathbf{T}_{1}^{*}}$ defining the space $\mathbf{T}_{1}$ (with base point $X_{1}$ ). Thus $\sigma_{\mu}$ descends to a holomorphic bijective map $\widehat{\sigma}_{\mu}$ of the space $\mathbf{T}_{1}$ onto itself, which implies the Teichmüller isometry

$$
\tau_{\mathbf{T}_{1}}\left(\phi_{\mathbf{T}_{1}}(\mu), \phi_{\mathbf{T}_{1}}(\nu)\right)=\tau_{\mathbf{T}_{1}}\left(\phi_{\mathbf{T}_{1}}(\mathbf{0}), \phi_{\mathbf{T}_{1}}\left(\sigma_{\mu}(\nu)\right), \quad \nu \in \operatorname{Belt}\left(f^{\mu}(\mathbb{D}), f^{\mu} \Gamma_{0}\left(f^{\mu}\right)^{-1}\right)_{1},\right.
$$

and similar admissible isometries for the Carathéodory and Kobayashi distances and their equality.

The case of infinitesimal metrics on $\widetilde{\mathbf{T}}$ is investigated in a similar way, which completes the proof of the theorem.

\section{Applications to geometric complex analysis}

3.1. General distortion theorem. Let $L$ be a bounded oriented quasicircle in the complex plane $\mathbb{C}$ separating the origin and the infinite point, with the interior and exterior domains $D$ and $D^{*}$ so that $0 \in D$ and $\infty \in D^{*}$. Let $\Sigma\left(D^{*}\right)$ denote the collection of uniavlent functions on $D^{*}$ with hydrodynamical normalization at $z=\infty$, and $\Sigma_{k}\left(D^{*}\right)$ be its subclass formed by functions $f^{\mu}$ admitting $k^{\prime}$-quasiconformal extensions to $D$ with $k^{\prime} \leq k$, which we additionally normalize by $f^{\mu}(0)=0$ or $f^{\mu}(1)=1$, and let $\Sigma^{0}\left(D^{*}\right)=\bigcup_{k} \Sigma_{k}\left(D^{*}\right)$.

Consider on this class a holomorphic (continuous and Gâteaux $\mathbb{C}$-differentiable) functional $J(f)$, which means that for any $f \in \Sigma^{0}\left(D^{*}\right)$ and small $t \in \mathbb{C}$,

$$
J(f+t h)=J(f)+t J_{f}^{\prime}(h)+O\left(t^{2}\right), \quad t \rightarrow 0,
$$

in the topology of uniform convergence on compact sets in $\mathbb{D}^{*}$. Here $J_{f}^{\prime}(h)$ is a $\mathbb{C}$-linear functional which is lifted to the strong (Fréchet) derivative of $J$ in the norms of both spaces $L_{\infty}(D)$ and $\mathbf{B}\left(D^{*}\right)$ (cf., e.g., [13, Ch. 3]). Any such functional $J$ is represented by a complex Borel measure on $\mathbb{C}$ and extends thereby to all holomorphic functions on $D^{*}$ (cf. [35]). 
We shall also use the notation $\widehat{J}(\mu)=J\left(f^{\mu}\right)$; the functional $\widehat{J}$ is holomorphic on the ball $\operatorname{Belt}(D)_{1}$.

In the class $\Sigma^{0}\left(D^{*}\right)$, we have a variational formula representing the maps with close Beltrami coefficients. This variation is represented via

$$
\omega=H^{\mu}(z)=z-\frac{1}{\pi} \iint_{D} \mu(\zeta) g(\zeta, z) d \xi d \eta+O\left(\|\mu\|_{\infty}^{2}\right) \quad\left(z \in D^{*}\right),
$$

with kernel

$$
g(\zeta, z)=\frac{1}{\zeta-z}+g_{1}(\zeta)
$$

where $g_{1}(\zeta)$ is a rational function determined by the normalization conditions, and the ratio $O\left(\|\mu\|_{\infty}^{2}\right) /\|\mu\|_{\infty}^{2}$ remains uniformly bounded on $\widehat{\mathbb{C}}$ as $\|\mu\|_{\infty} \rightarrow 0$ (see, e.g., [15]). In particular, in our case,

$$
g(\zeta, z)=\frac{1}{\zeta-z}-\frac{1}{1-z}
$$

and a neighborhood of the identity map id is filled by the maps

$$
\begin{aligned}
f^{\mu}(z) & =z-\frac{1}{\pi} \iint_{\mathbb{D}} \mu(\zeta)\left(\frac{1}{\zeta-z}-\frac{1}{\zeta-1}\right) d \xi d \eta+O\left(\|\mu\|^{2}\right) \\
& =z-\frac{z-1}{\pi} \iint_{\mathbb{D}} \frac{\mu(\zeta) d \xi d \eta}{(\zeta-1)(\zeta-z)}+O\left(\|\mu\|^{2}\right) \quad \text { as } \quad\|\mu\| \rightarrow 0 .
\end{aligned}
$$

This yields the corresponding functional derivative of $J$ at the origin

$$
\psi_{0}(z)=J_{\text {id }}^{\prime}(g(\mathrm{id}, z))
$$

The notation for the kernel $g(\mathrm{id}, z)$ applied here and below relates to the fact that after the change $\zeta=f^{\nu}(Z)$ with $\nu$ running over the set $X$ of Beltrami coefficients from Belt( $(\mathbb{C})_{1}$ preserving the domain $D$, one obtains for any fixed finite $z \in D^{*}$ a continuous (and real analytic) functional $\check{g}(\nu)=g\left(f^{\nu}, \cdot\right): X \rightarrow \mathbb{C}$.

We assume that the functional derivative (3.2) is meromorphic on $\mathbb{C}$ and has in the domain $D$ only a finite number of the simple poles (hence $\psi_{0}$ is integrable over $D$ ). This holds, in particular, for the general distortion functionals of the form

$$
J(f):=J\left(f(a) ; f\left(z_{1}\right), f^{\prime}\left(z_{1}\right), \ldots, f^{\left(\alpha_{1}\right)}\left(z_{1}\right) ; \ldots ; f\left(z_{p}\right), f^{\prime}\left(z_{p}\right), \ldots, f^{\left(\alpha_{p}\right)}\left(z_{p}\right)\right)
$$

with grad $\widehat{J}(\mathbf{0}) \neq 0$, where $z_{1}, \ldots, z_{p}$ are distinct fixed points in $D^{*}$ with assigned orders $\alpha_{1}, \ldots, \alpha_{p}$ and $a$ is a fixed point in the domain of quasiconformality $D$. In this case, one derives, representing $f$ by (3.1), that $\psi_{0}$ is a rational function

$$
\widehat{J}_{\mathrm{id}}^{\prime}(g(\mathrm{id}, z))=\frac{\partial \widehat{J}(\mathbf{0})}{\partial \omega} g(z, a)+\left.\sum_{j=1}^{p} \sum_{s=0}^{\alpha_{j}-1} \frac{\partial \widehat{J}(\mathbf{0})}{\partial \omega_{j, s}} \frac{d^{s}}{d \zeta^{s}} g(w, \zeta)\right|_{w=z, \zeta=z_{s}},
$$

where $\omega=f(a), \omega_{j, s}=f^{(s)}\left(z_{j}\right)$.

The above theorems on complex geodesics provide a general distortion theorem which sheds light on underlying features and, on the other hand, implies the sharp explicit bounds.

Theorem 3.1. (i) For any functional $J$ of type (3.3) whose range domain $J\left(\Sigma^{0}\left(D^{*}\right)\right)$ has more than two boundary points, there exists a number $k_{0}(J)>0$ such that for all $k \leq k_{0}(J)$, we have the sharp bound

$$
\max _{\|\mu\| \leq k}\left|J\left(f^{\mu}\right)-J(\mathrm{id})\right| \leq \max _{|t|=k}\left|J\left(f^{t\left|\psi_{0}\right| / \psi_{0}}\right)-J(\mathrm{id})\right|
$$


in other words, the values of $J$ on the ball $\operatorname{Belt}(D)_{k}=\left\{\mu \in \operatorname{Belt}(D)_{1}:\|\mu\|_{\infty} \leq k\right\}$ are placed in the closed disk $\mathbb{D}\left(J(\mathrm{id}), M_{k}\right)$ with center at $J(\mathrm{id})$ and radius $M_{\kappa}=\max _{|t|=k}\left|J\left(f^{t\left|\psi_{0}\right| / \psi_{0}}\right)-J(\mathrm{id})\right|$. The equality occurs only for $\mu=t\left|\psi_{0}\right| / \psi_{0}$ with $|t|=k$.

(ii) Conversely, if a functional $J$ is bounded via (3.4) for $0<k \leq k_{0}(J)$ with some $k_{0}(J)>0$, then up to rescaling (multiplying $J$ by a positive constant factor),

$$
J\left(f^{\mu}\right)=\mathcal{F}\left(S_{f^{\mu}}\right)+O\left(\|\mu\|_{\infty}^{2}\right) \quad \text { as } \quad\|\mu\|_{\infty} \rightarrow 0,
$$

where $\mathcal{F}$ is holomorphic on $\mathbf{T}_{1}$ and its renormalization $\widetilde{\mathcal{F}}(\varphi)=\mathcal{F}(\varphi) / \sup _{\varphi \in \mathbf{T}_{1}}|\mathcal{F}(\varphi)|$ is the defining map for the disk $\mathbb{D}\left(\mu_{0}\right)$ as a $c_{\mathbf{T}_{1}}$-geodesic in the space $\mathbf{T}_{1}$ with the base point representing the punctured quasidisk $D \backslash\{a\}$.

Proof. For certain specific functionals $J$, such a theorem was proved in [17, [19], 20]. The proof in the general case follows the same lines and essentially involves Theorem 1.2. Without loss of generality, one can assume that $J(\mathrm{id})=0$.

First we mention the important facts concerning the projections of norm 1 in Banach spaces given in the auxiliary Lemmas 4.1 and 4.2, the proof of which can be found in [5].

Let $V$ be a complex Banach space with norm $\|\cdot\|$ differentiable on $V \backslash\{0\}$, and suppose that

$$
A(v, w)=\lim _{t \rightarrow 0} \frac{\|v+t w\|-\|v\|}{t} \text { for all } v \in V \backslash\{0\}, w \in V ;
$$

for every fixed $v \neq 0$ it is a bounded linear functional on $V$.

Lemma 3.2. Let $W$ be a non-trivial closed (complex) subspace of $V$, and let $W^{\prime}$ be the closed subspace

$$
W^{\prime}=\{w \in V: A(v, w)=0 \quad \text { for all } v \in W \backslash\{0\} .
$$

There is a projection $P$ of norm 1 from $V$ onto $W$ if and only if $W^{\prime}$ is a complementary subspace to $W$, that is $W \oplus W^{\prime}=V$. Further, if $P$ exists, it is unique and its kernel is $W^{\prime}$.

The next lemma is a straightforward modification of the corresponding lemmas of Royden [33] and of Earle and Kra [5].

Let $a_{1}, a_{2}, \ldots, a_{n}$ be distinguished points of a domain $D \subseteq \mathbb{C}$, and let $\varphi$ and $\psi$ be $L_{1}$ functions on $D$, holomorphic and nonzero for $z \in D \backslash\left\{a_{1}, a_{2}, \ldots, a_{n}\right\}$. Denote the orders of the functions $\varphi$ and psi at the points $a_{j}$ by $\alpha_{j}$ and $\beta_{j}$, respectively $\left(\alpha_{j}, \beta_{j} \geq-1\right)$. For real $t$, we consider the function

$$
h(t)=\iint_{D}|\varphi(z)+t \psi(z)| d x d y .
$$

Lemma 3.3. The function $h(t)$ is differentiable near $t=0$, and

$$
h^{\prime}(0)=\operatorname{Re} \iint_{D} \psi(z) \frac{\overline{\varphi(z)}}{\varphi(z)} d x d y .
$$

Moreover, if $\alpha_{j} \leq 2 \beta_{j}+1$ for all $j=1, \ldots, n$, then the second derivative $h^{\prime \prime}(0)$ exists. If $\alpha_{j}>2 \beta_{j}+1$ for some $j$, then

$$
h(t)=h(0)+t h^{\prime}(0)+\sum_{1}^{n} c_{j} \delta_{j}(t)+o\left(\max _{j} \delta_{j}(t)\right),
$$

where all the $c_{j}$ are positive constants, and

$$
\delta_{j}= \begin{cases}t^{2} \log (1 /|t|), & \alpha_{j}=2 \beta_{j}+2, \\ |t|^{1+\left(2+\beta_{j}\right) /\left(\alpha_{j}-\beta_{j}\right)}, & \alpha_{j}>2 \beta_{j}+2 .\end{cases}
$$


Note that the equality (3.6) is obtained by applying Lebesgue's theorem of dominant convergence, which is possible in view of the obvious inequality

$$
\left|\frac{|\varphi+t \psi|-|\varphi|}{t}\right| \leq|\psi|
$$

As for the relations (3.7) and (3.8), for any close subdomain $E \Subset D \backslash\left\{a_{1}, \ldots,, a_{n}\right\}$ the integral $\iint_{E}|\varphi+t \psi| d x d y$ is an infinitely differentiable function of $t$, hence the derivation of these relations reduces to estimating the contributions of integrals of the form

$$
I_{j}(t)=\iint\left(\left|z-a_{j}\right|^{\alpha}+t \omega_{j}(z)|-| z-\left.a_{j}\right|^{\alpha}-t \operatorname{Re}\left[\omega_{j}(z)\left(\frac{z-a_{j}}{\mid z-a_{j}}\right)^{\alpha}\right]\right) d x d y
$$

over sufficiently small disks $\left\{\left|z-a_{j}\right|<r\right\}$.

Now let $f_{0}$ be any function in $\Sigma_{k}\left(D^{*}\right)$ maximizing $|J|$ over $\Sigma_{k}\left(D^{*}\right)$ (the existence of such $f_{0}$ follows from compactness). We may assume that its Beltrami coefficient $\mu_{f_{0}}$ is extremal in its class, i.e.,

Suppose that

$$
\left\|\mu_{f_{0}}\right\|_{\infty}=\inf \left\{\|\mu\|_{\infty} \leq k: f^{\mu}\left|D^{*}=f_{0}\right| D^{*}\right\} .
$$

$$
\mu_{f_{0}} \neq t \mu_{0} \quad \text { for some } t \text { with }|t|=k \text {, }
$$

where

$$
\mu_{0}=\left|\varphi_{0}\right| / \varphi_{0}
$$

We show that for small $k$ this leads to a contradiction.

First we establish the following important property of extremal maps which sheds light to the main underlying features. Pick in $A_{1}(D \backslash\{a\})$ the functions

$$
\omega_{p}(z)=z^{p}-1-\psi_{0}(z), \quad p=1,2, \ldots \quad ; \quad \rho_{a}(z)=\frac{a-1}{(z-1)(z-a)} .
$$

Lemma 3.4. For sufficiently small $k \leq k_{0}(J)$, the extremal Beltrami coefficient $\mu_{f_{0}}$ is orthogonal to all functions (3.11), i.e.,

$$
\left\langle\mu_{f_{0}}, \psi_{p}\right\rangle_{D}=0 .
$$

Proof. Note that from (3.1) and (3.2),

$$
\widehat{J}(\mu)=-\frac{1}{\pi}\left\langle\mu, \varphi_{0}\right\rangle_{D}+O\left(\|\mu\|^{2}\right),
$$

and hence,

$$
\left|\widehat{J}\left(\mu_{f_{0}}\right)\right|=\max _{\|\mu\| \leq k}|\widehat{J}(\mu)|=\frac{k}{\pi} \iint_{D}\left|\varphi_{0}\right| d x d y+O\left(k^{2}\right)=\left\|J_{\text {id }}^{\prime}\right\| k+O\left(k^{2}\right),
$$

where $\left\|J_{\text {id }}^{\prime}\right\|$ is defined by (3.1). Consider the auxiliary functional

$$
\widehat{J}_{p}(\mu)=\widehat{J}(\mu)+\xi\left\langle\mu, \psi_{p}\right\rangle_{D},
$$

where $p$ is fixed and $\xi \in \mathbb{C}$. Then, similar to (3.14),

$$
\max _{\|\mu\| \leq k}\left|\widehat{J}_{p}(\mu)\right|=\frac{k}{\pi} \iint_{D}\left|\varphi_{0}(z)+\xi \psi_{p}(z)\right| d x d y+O\left(\|\mu\|^{2}\right)
$$

and the remainder term estimate is independent of $p$.

Using the properties of the norm

$$
h_{p}(\xi)=\iint_{D}\left|\varphi_{0}(z)+\xi \psi_{p}(z)\right| d x d y
$$


deduced from Lemmas 3.2 and 3.3 one obtains from (3.14), (3.16) that for small $\xi$ there should be $h_{p}^{\prime}(0)=0$, and

$$
\max _{\|\mu\| \leq k}\left|\widehat{J}_{p}(\mu)\right|=\max _{\|\mu\| \leq k}\left|\widehat{J}\left(\mu_{f_{0}}\right)\right|+k o_{p}(\xi)+O_{p}\left(k^{2} \xi\right)+O\left(k^{2}\right) .
$$

Now fix $k$ and consider the classes $\Sigma_{\tau k}\left(D^{*}\right), \tau<1$. Then from (3.17) we have

$$
\max _{\|\mu\| \leq \tau k}\left|\widehat{J}_{p}(\mu)\right|=\max _{\|\mu\| \leq \tau k}|\widehat{J}(\mu)|+\tau o_{p}(\xi)+O_{p}\left(\tau^{2} \xi\right)+O\left(\tau^{2}\right) .
$$

On the other hand, we have from (3.15) that as $\xi \rightarrow 0, \tau \rightarrow 0$, there should be

$$
\begin{aligned}
\left|\widehat{J}_{p}\left(\tau \mu_{f_{0}}\right)\right| & =\left|\widehat{J}\left(\tau \mu_{f_{0}}\right)\right|+\operatorname{Re} \frac{\overline{\widehat{J}\left(\tau \mu_{f_{0}}\right)}}{\widehat{\widehat{J}\left(\tau \mu_{f_{0}}\right)}} \tau \xi\left\langle\mu_{f_{0}}, \psi_{p}\right\rangle+O\left(\tau^{2} \xi^{2}\right) \\
& =\left|\widehat{J}\left(\tau \mu_{0}\right)\right|+\tau|\xi|\left\langle\mu_{f_{0}}, \psi_{p}\right\rangle_{\mathbb{D}}+O\left(\tau^{2} \xi^{2}\right)
\end{aligned}
$$

with suitable choices of $\xi \rightarrow 0$. Comparison with (3.18) implies the desired equalities (3.12), completing the proof of the lemma.

We now apply a geodesic holomorphic map $\mathcal{F}: \mathbf{T}_{1} \rightarrow \mathbb{D}$ from Theorem 1.2 defining the disk $\mathbb{D}\left(\mu_{0}\right)$ as $c_{\mathbf{T}_{1}}$-geodesic; it determines a hyperbolic isometry between this disk and $\mathbb{D}$. We lift this map onto $\operatorname{Belt}(\mathbb{D})_{1}$ by $\Lambda(\mu)=\mathcal{F} \circ \phi_{\mathbf{T}_{1}}(\mu)$ getting a holomorphic map of this ball onto the disk. The differential of $\Lambda$ at $\mu=\mathbf{0}$ is a linear operator $P: L_{\infty}(\mathbb{D}) \rightarrow L_{\infty}(\mathbb{D})$ of norm 1 which is represented in the form

$$
P(\mu)=\beta\left\langle\mu, \psi_{0}\right\rangle_{D} \mu_{0} .
$$

Let $P\left(\mu_{f_{0}}\right)=\alpha(\kappa) \mu_{0}$. Since, by assumption, $f_{0}$ is not equivalent to $f^{t_{0} \mu_{0}}$ with $\left|t_{0}\right|=\kappa$, we have

$$
\left\{\Lambda\left(\frac{t}{\kappa} \mu_{f_{0}}\right):|t|<1\right\} \varsubsetneqq\{|t|<1\} .
$$

Thus, by Schwarz's lemma,

$$
|a(k)|<k
$$

Now consider the function

$$
\nu_{0}=\mu_{f_{0}}-\alpha(k) \mu_{0}
$$

which is not equivalent to zero, due to our assumption (4.5). We show that $\nu_{0}$ annihilates integrable holomorphic functions on $\mathbb{D}$.

First of all, we have

$$
\left\langle\nu_{0}, \varphi\right\rangle_{D}=0
$$

for all $\varphi$ from the subspace $W^{\prime}=\left\langle\psi_{p}\right\rangle$ of $A_{1}(D)$ spanned by $(3.11)$, since $\left\langle\mu_{f_{0}}, \varphi\right\rangle_{\mathbb{D}}=0$ by Lemma 3.4 and $\left\langle\mu_{0}, \varphi\right\rangle_{D}=0$ by Lemma 2.1 applied to one-dimensional subspace $W=\left\{\lambda \varphi_{0}: \lambda \in \mathbb{C}\right\}$. To establish that

consider the conjugate operator

$$
\left\langle\nu_{0}, \varphi_{0}\right\rangle_{\mathbb{D}}=0
$$

$$
P^{*}(\varphi)=\left\langle\mu_{0}, \varphi\right\rangle_{D} \varphi_{0}
$$

which maps $L_{1}(D)$ into $L_{1}(D)$ and fixes the subspace $W=\left(\omega_{p}, \rho_{a}\right)$ of $A_{1}(D \backslash\{a\})$ spanned by functions (3.11).

The definition of $\nu_{0}$ implies $P\left(\nu_{0}\right)=0$, thus

$$
\left\langle\nu_{0}, \varphi_{0}\right\rangle_{D}=\lambda\left\langle\nu_{0}, P^{*} \nu_{0}\right\rangle_{D}=\left\langle P \nu_{0}, \varphi_{0}\right\rangle_{D}=0 .
$$

Since the functions $\varphi_{0}, \psi_{p}, p=0,1, \ldots$, form a complete set in the space $A_{1}(D \backslash\{a\})$, we have proved that $\nu_{0}$ is orthogonal to all $\varphi \in A_{1}(D \backslash\{a\})$, i.e., belongs to the set

$$
A_{1}(D \backslash\{a\})^{\perp}=\left\{\mu \in L_{\infty}(D):\langle\mu, \varphi\rangle_{D}=0 \quad \text { for all } \varphi \in A_{1}(D \backslash\{a\})\right\} .
$$


Now we use the well-known properties of extremal quasiconformal maps (see e.g., 8], [15], 31]). First of all, since $\mu_{f_{0}}$ is extremal for $f_{0}$,

$$
\left\|\mu_{f_{0}}\right\|_{\infty}=\inf \left\{\left|\left\langle\mu_{f_{0}}, \varphi\right\rangle_{D}\right|: \varphi \in A_{1}(D \backslash\{a\}),\|\varphi\|=1\right\} ;
$$

moreover, by Hamilton-Krushkal-Reich-Strebel theorem such an equality is necessary and sufficient for $\mu \in \operatorname{Belt}(D)_{1}$ to be extremal for $f^{\mu}$. Hence, if $f_{0}$ is extremal in its class, then for any $\nu \in$ $A_{1}(D \backslash\{a\})^{\perp}$,

$$
k=\left\|\mu_{f_{0}}\right\|_{\infty}=\inf \left\{\mid\left\langle\mu_{f_{0}}+\nu, \varphi\right\rangle_{D}: \varphi \in A_{1}(D \backslash\{a\}),\|\varphi\|=1\right\} \leq\left\|\mu_{f_{0}}+\nu\right\|_{\infty},
$$

which implies

$$
k \leq\left\|\mu_{f_{0}}-\nu_{0}\right\|_{\infty}=\left\|\alpha(k) \mu_{0}\right\|_{\infty}=\alpha(k),
$$

in contradiction to (3.19). This proves the first part of the theorem.

The proof of the converse part (ii)) is much simpler. Lifting the original functional $J$ with $J(\mathrm{id})=0$ to

$$
I(\mu)=\pi^{-1} \circ J\left(f^{\mu}\right): \operatorname{Belt}(D)_{1} \rightarrow \mathbb{D},
$$

where $\pi$ is a holomorphic universal covering of the domain $V(J)=J\left(\Sigma^{0}\left(D^{*}\right)\right)$ by a disk $\mathbb{D}_{a}=$ $\{|z|<a\}$ with $\pi(0)=0, \pi^{\prime}(0)=1$, one obtains

$$
\pi(\zeta)=\zeta+O\left(\zeta^{2}\right), \quad \zeta \rightarrow 0
$$

with uniform estimate of the remainder for $|\zeta|<\left|\zeta_{0}\right|$, which implies the asymptotic equality (3.5). The covering functional $I$ generates a holomorphic map $\widetilde{I}: \mathbf{T}_{1} \rightarrow \mathbb{D}$ so that $I=\widetilde{I} \circ \phi_{\mathbf{T}_{1}}$, and by (3.4),

$$
\max _{k\left(f^{\mu}\right) \leq k}\left|I\left(f^{\mu}\right)\right|=k \quad \text { for } 0<k<k_{1}(I) .
$$

Restricting the covering map $\widetilde{I}$ to the extremal disk $\left\{\phi_{\mathbf{T}_{1}}\left(t \mu_{0}^{*}\right):|t|<1\right\} \subset \mathbf{T}_{1}$ (where $\left.\mu_{0}^{*}=\left|\psi_{0}\right| / \psi_{0}\right)$, one derives by Schwarz's lemma that $\widetilde{I}\left(\phi_{\mathbf{T}_{1}}\left(t \mu_{0}^{*}\right)\right) \equiv t$. Thus the inverse to this map must be $c_{\mathbf{T}^{-}}$ geodesic, which completes the proof of Theorem 3.1.

3.2. A lower estimate for the bound $k_{0}(J)$. If the functional $J$ is bounded on the whole class $\Sigma^{0}\left(D^{*}\right)$, and $J(\mathrm{id})=0, \operatorname{grad} J(\mathrm{id}) \neq 0$, one can derive from the proof of Theorem 3.1 similar to [17] an effective lower bound for $k_{0}(J)$ :

$$
k \leq k_{0}(J)=\frac{\left\|J_{\text {id }}^{\prime}\right\|}{\left\|J_{\text {id }}^{\prime}\right\|+M(J)+1},
$$

where

$$
\left\|J_{\text {id }}^{\prime}\right\|=\frac{1}{\pi}\left\|\psi_{0}\right\|_{1}, \quad M(J)=\sup _{\Sigma^{0}\left(D^{*}\right)}|J(f)| .
$$

Theorem 3.1 provides new various explicit estimates controlling the distortion in both conformal and quasiconformal domains simultaneously. A similar theorem is valid also for univalent functions on bounded quasidisks $D$, for example, for the canonical class $S_{k}(D)$ of univalent functions in $D$ normalized by $f(z)=z+c_{2} z^{2}+\ldots$ near the origin (provided that $z=0 \in D$ ) and admitting $k$-quasiconformal extensions to $\widehat{\mathbb{C}}$ which preserve the infinite point. Earlier only very special results have been established here (see [12], [15], [25], [26]).

3.3. Remark. It is essential that the distinguished point $a$ is inner, for $a \in \partial D$ the estimate (3.3) can fail and an additional remainder term $O\left(k^{2}\right)$ can appear (see, for example, Kühnau's description of the domain of values of $f(1)$ on $\Sigma_{k}$ in [22, Part 2]). 


\section{NEW PHENOMENA}

4.1. Rigidity of extremals. The intrinsic connection between the extremals of the distortion functionals on functions with quasiconformal extensions and complex geodesics causes surprising phenomena which do not appear in the classical theory concerning all univalent functions. The differences arise from the fact that in problems for the functions with quasiconformal extensions the extremals belong to compact subsets of $\Sigma^{0}\left(D^{*}\right)$ (or in other functional classes), while the maximum on the whole class is attained on the boundary functions.

The following consequence of Theorem 3.1, part (ii), provides strong rigidity of extremal maps.

Corollary 4.1. In any class of univalent functions with $\kappa$-quasiconformal extension, no function can be simultaneously extremal for different holomorphic functionals (3.4) unless these functionals have equal 1-jets at the origin.

4.2. Example: the coefficient problem for functions with quasiconformal extensions. We mention here an improvement in estimating the Taylor coefficients. Though the Bieberbach conjecture for the canonical class $S$ of univalent functions $f(z)=z+\sum_{2}^{\infty} a_{n} z^{n}$ in $\mathbb{D}$ has already been proved by de Brange's theorem, the old coefficient problem remains open for univalent functions in the disk with quasiconformal extensions. The problem was solved by the author for the functions with sufficiently small dilatations.

Denote by $S_{k}(\infty)$ and $S_{k}(1)$ the classes of $f \in S$ admitting $k$-quasiconformal extensions $\widehat{f}$ to $\widehat{\mathbb{C}}$ normalized by $\widehat{f}(\infty)=\infty$ and $\widehat{f}(1)=1$, respectively. Let

$$
f_{1, t}(z)=\frac{z}{(1-t z)^{2}}, \quad|z|<1, \quad|t|<1 .
$$

This function can be regarded as a quasiconformal counterpart of the well-known Koebe function which is extremal for many functionals on $S$ (see, e.g. [10], [30]).

As a special case of Theorem 3.1, we have a complete solution of the Kühnau-Niske problem:

Theorem 4.2. [17] For all $f \in S_{k}(\infty)$ and all $k \leq 1 /\left(n^{2}+1\right)$,

$$
\left|a_{n}\right| \leq 2 \kappa /(n-1),
$$

with equality only for the functions

$$
f_{n-1, t}(z)=f_{1, t}\left(z^{n-1}\right)^{1 /(n-1)}=z+\frac{2 t}{n-1} z^{n}+\ldots, \quad n=3,4, \ldots ; \quad|t|=k .
$$

The estimate (4.2) also holds in the classes $S_{k}(1)$ with the same bound for $k$.

Until now, no estimates have been obtained for arbitrary $k<1$, unless $n=2$; in the last case, $\left|a_{2}\right| \leq 2 k$ with equality for the function (4.1) when $|t|=k$.

The rigidity provided by Corollary 4.1 yields that the function (4.1) cannot maximize $\left|a_{n}\right|$ in $S_{k}(\infty)$ even for one $k<1$, unless $n=2$. Hence, for all $k<1$,

$$
\max _{f \in S_{k}(\infty)}\left|a_{n}\right|>n k^{n-1} \quad(n \geq 3) .
$$

For $n=3$, this estimate was established also by Kühnau-Niske [28] using elliptic integrals. 
Comparing the coefficients $a_{n}$ of $f_{1, t}$ and $f_{n-1, t}$, one derives from (4.2) and (4.4) the rough bounds for the maximal value $k_{n}$ of admissible $\kappa$ in (4.2):

$$
\frac{1}{n^{2}+1} \leq k_{n}<\left[\frac{2}{n(n-1)}\right]^{1 /(n-2)} .
$$

4.3. Over-normalized functions. Another remarkable thing in the distortion theory for univalent functions with quasiconformal extension concerns over-determined normalizations what reveal the intrinsic features of quasiconformality. The variational problems for such classes are originated in the 1960s from several points of view. The results were obtained mainly in terms of inverse extremal functions $f_{0}^{-1}$ (see [15], 2], 9], 32, 36]). But until now no explicit estimates have been established. We establish here some general explicit bounds for the functions univalent in the generic disks using complex geodesics in the space $\mathbf{T}_{1}$.

Assume that $z=1$ lies on the common boundary of $D$ and $D^{*}$ which separates the points 0 and $\infty$ and denote by $\Sigma^{0}\left(D^{*}, 1\right)$ the class of univalent functions in $D^{*}$ with quasiconformal extensions across $L$ which satisfy

$$
f(z)=z+\text { const }+O(1 / z) \text { near } z=\infty ; \quad f(1)=1,
$$

and by $\Sigma_{\kappa}\left(D^{*}, 1\right)$ its subclasses consisting of functions with $\kappa$-quasiconformal extensions. Fix in $D^{*}$ a finite collection of points $e_{1}, \ldots, e_{m-1}, m>1$, and a point $e_{m}$ in the complementary domain D. Put

$$
e=\left(e_{1}, \ldots, e_{m}\right) .
$$

We associate with this fixed point set the following subspaces of $L_{1}(\mathbb{C})$ : the span $\mathcal{L}(e)$ of rational functions

$$
\rho_{s}(z)=\frac{e_{s}-1}{(z-1)\left(z-e_{s}\right)} \quad s=1, \ldots, m
$$

the space $A_{1}\left(D_{e}\right)$ of integrable holomorphic functions in the punctured domain $D_{e}=D \backslash\left\{e_{m}\right\}$, and

$$
\mathcal{L}_{0}=\mathcal{L}(e) \bigoplus\left\{c \psi_{0}: c \in \mathbb{C}\right\},
$$

where $\psi_{0}=J_{\text {id }}^{\prime}(g(\mathrm{id}, \cdot))$ for $g(w, \zeta)$ given above. Denote by $\Sigma_{k}\left(D^{*}, 1, e\right)$ the collection of $f \in$ $\Sigma_{k}\left(D^{*}, 1\right)$ satisfying

$$
f\left(e_{s}\right)=e_{s}, \quad s=1, \ldots, m,
$$

and let $\Sigma^{0}\left(D^{*}, 1, e\right)=\bigcup_{k} \Sigma_{k}\left(D^{*}, 1, e\right)$.

Note that these classes with over-determined normalization contain nontrivial maps $f^{\mu} \neq$ id for any $k<1$, which is insured by Lemma 2.3 .

Our aim is to estimate on such over-normalized classes the functionals

$$
J(f)=J\left(f\left(z_{1}\right), f^{\prime}\left(z_{1}\right), \ldots, f^{\left(\alpha_{1}\right)}\left(z_{1}\right) ; \ldots ; f\left(z_{p}\right), f^{\prime}\left(z_{p}\right), \ldots, f^{\left(\alpha_{p}\right)}\left(z_{p}\right)\right),
$$

with $\operatorname{grad} J(\mathrm{id}) \neq 0$, controlling the distortion on the domain of conformality. To simplify the notations, assume that $J(\mathrm{id})=0$ and $|J(f)| \leq 1$ on $\Sigma\left(D^{*}\right)$.

Now one can use only conditional quasiconformal variations whose Beltrami coefficients are orthogonal to the rational quadratic differentials (4.5) corresponding to the fixed points. So the general variational technique does not work.

From geometric point of view, the equations

$$
f\left(e_{1}\right)=e_{1}, \ldots, f\left(e_{m}\right)=e_{m}
$$

define an analytic set $\mathcal{Z}$ of codimension $m$ in the Banach domain modeling the universal Teichmüller space $\mathbf{T}$. The generic geodesic disks

$$
\left\{\phi_{\mathbf{T}}(t \mu): \mu \in \operatorname{Belt}(\mathbb{D})_{1}, t \in \mathbb{D}\right\}
$$


defining the distortion estimates in Theorem 3.1 can have at most a finite number common points with this set $\mathcal{Z}$. This shows also that the classes $\Sigma_{k}\left(D^{*}, 1, e\right)$ contains the maps with dilatations close to 1 .

4.4. Sharp explicit bounds for small dilatations. The following distortion bounds involve $L_{1}$-distance between the functional derivative $\psi_{0}$ and span $\mathcal{L}(e)$; these bounds hold also in the case when some fixed points $e_{s} \in \partial D$.

Theorem 4.3. For every functional (4.7) and any finite set e of fixed points defined above, there exists a positive number $k_{0}(J, e)<1$ such that for all $k \leq k_{0}(J, e)$, we have for any function $f \in \Sigma_{k}\left(D^{*}, 1, e\right)$ the sharp bound

$$
\max _{\|\mu\| \leq k}\left|J\left(f^{\mu}\right)\right|=\max _{|t|=\kappa}\left|J\left(f^{t\left|\psi_{e}\right| / \psi_{e}}\right)\right|=d \kappa+O\left(\kappa^{2}\right)
$$

with uniformly bounded ratio $O\left(\kappa^{2}\right) / \kappa^{2}$. Here $\kappa=\kappa(k)<k$ is the bound for Teichmüller norms of $f \in \Sigma_{k}\left(D^{*}, 1, e\right)$ (i.e, regarding these $f$ as the functions of $\Sigma\left(D^{*}, 1\right)$ with omitted restrictions $(4.8))$,

$$
\psi_{e}=\psi_{0}+\sum_{1}^{m} \xi_{s} \rho_{s}
$$

with some constants $\xi_{1}, \ldots, \xi_{m}$ and

$$
d=\inf _{\psi \in \mathcal{L}(e)}\left\|\psi_{0}-\psi\right\|_{1}=
$$

These constants are determined (not necessary uniquely) from the equalities

$$
\left\langle\left|\psi_{e}\right| / \psi_{e}, \psi\right\rangle_{D}=0 \quad \text { for all } \quad \psi \in \mathcal{L}(e) ; \quad\left\langle\left|\psi_{e}\right| / \psi_{e}, \psi_{0}\right\rangle_{D}=d
$$

and from condition that the norm of functional $\left\langle f_{e}, \rho\right\rangle$ on $L_{1}$ equals 1 .

Proof. In view of Theorem 3.1, the functional $J$ can be regarded as a linear functional on the span generated in $A(D)$ by $\psi_{0}$. Since the points $e_{s}$ are preserved, the variation of $J$ must be orthogonal to $\mathcal{L}(e)$.

By the Hahn-Banach theorem, there exists a linear functional $l$ on $L_{1}(D)$ such that

$$
l(\psi)=0, \quad \psi \in \mathcal{L}(e) ; \quad l\left(\psi_{0}\right)=d,
$$

and

$$
\|l\|_{L_{1}(D)}=\|l\|_{\mathcal{L}_{0}}=1
$$

and this norm is minimal on the spaces $\mathcal{L}_{0} \subset A_{1}\left(D \backslash\left\{e_{m}\right\}\right) \subset L_{1}(D)$. It is generated by $l(\psi)=t d$ for $\psi \in \mathcal{L}_{0}$ and extended to $L_{1}(D)$ preserving the norm. Hence, for any other linear functional $\tilde{l}$ on $L_{1}(D)$ satisfying (4.13) must be $\|\widetilde{l}\|_{\mathcal{L}_{0}} \geq 1$. We need to take $\kappa l(\psi)$.

The functional $l$ is represented on $L_{1}(D)$ via

$$
l(\psi)=\iint_{D} \nu_{0}(z) \psi(z) d x d y, \quad \psi \in L_{1},
$$

with some $\nu_{0} \in L_{\infty}(D)$ so that

$$
\iint_{D} \nu_{0}(z) \psi(z) d x d y=0, \quad \psi \in \mathcal{L}(e) ; \quad \iint_{D} \nu_{0}(z) \psi_{0}(z) d x d y=d .
$$

Since the norm of $l$ on the widest space $L_{1}(D)$ is attained on its subspace $\mathcal{L}(e)$, the function $\nu_{0}$ is of the form $\nu_{0}(z)=\left|\psi_{e}(z)\right| / \psi_{e}(z)$ with integrable holomorphic $\psi_{e}$ on $D \backslash\left\{e_{m}\right\}$ given by (4.10). 
After extending $\nu_{0}$ by zero to $D^{*}$, which yields an extremal Beltrami coefficient $\nu_{0} \in \operatorname{Belt}(D)_{1}$ for our holomorphic functional $J$ (with possible pole at the point $e_{m}$ ), one can represent the map $f^{t \nu_{0}}$ by (4.10) getting from the second equality in (4.14) and from indicated minimality of $\|l\|$ the estimate (4.12).

However, this $k$-quasiconformal map can move the fixed points $e_{s}$ to $f_{0}\left(e_{s}\right)=e_{s}+O\left(k^{2}\right)=$ $e_{s}+O\left(\kappa(k)^{2}\right)$, where $f_{0}=f^{\nu_{0}}$. For correction, one needs to apply additional $O\left(k^{2}\right)$-quasiconformal variation $h_{0}$ by Lemma 4.3 .

It can be shown, using the uniqueness of Teichmüller extremal maps generated by integrable holomorphic quadratic differential that this $\psi_{e}$ is unique in $A_{1}\left(D \backslash\left\{e_{m}\right\}\right)$.

The assertion on uniform bound for the remainder in (4.9) follows from the general distortion results for quasiconformal maps (see [15]), completing the proof.

Remark 1. The assumption $f(1)=1$ can be replaced by $f(0)=0 \in D$; then the fixed point $e_{m}$ must be chosen to be distinct from the origin.

4.5. Some applications. In the case when the set $e$ consists of only one point $e_{m}=\zeta_{0}$ located in the domain $D$ of quasiconformality (i.e., the functions $f \in \Sigma^{0}\left(D^{*}, 1, e\right)$ are normalized hydrodynamically at $\infty$ and $\left.f(1)=1, f\left(\zeta_{0}\right)=\zeta_{0}\right)$ one can essentially improve Theorem 4.4 getting a global distortion bound of type Theorem 3.1.

Then the equation $f\left(\zeta_{0}\right)=\zeta_{0}$ defines in the space $\mathbf{T}_{1}$ an analytic set $\mathcal{Z}_{0}$ of codimension 1 , and the generic geodesic disks joining the origin of $\mathbf{T}_{1}$ with the points of $\mathcal{Z}_{0}$ are located partially outside of $\mathcal{Z}_{0}$. Take for the points $X=\left(S_{f}, z\right) \in \mathcal{Z}_{0}$ the nonsingular holomorphic disks $\Delta=h(\mathbb{D})$ in $\mathbf{T}_{1}$ joining these points with the origin and pull back the hyperbolic metric $\lambda_{\mathbb{D}}$ onto such disks using the functions $\widehat{J} \circ h: \mathbb{D} \rightarrow \mathbb{D}$, where $\widehat{J}$ denotes the lifting of the functional $J$ onto the space $\mathbf{T}_{1}$. This yields a subharmonic metric $\lambda_{J}$ on $\Delta$ of curvature -4 at its noncritical points. In particular, there is a domain $\Omega \subset \Delta$ containing the origin filled by the points of $\mathcal{Z}_{0}$. Comparing this metric with the infinitesimal Finsler metric $F_{\mathbf{T}}\left(S_{f}, v\right)$ (which defines the Teichmüller dilatation $\kappa=\tanh \tau_{\mathbf{T}}$ ) similar to part $\mathbf{3}^{0}$ of the proof of Theorem 3.1, one derives the following bound:

Proposition 4.4. For $0<k<k_{0}\left(J, \zeta_{0}\right) \leq 1$,

$$
\max \left\{\mid J\left(f^{\mu}\right): f^{\mu} \in \Sigma\left(D^{*}, 1, e\right),\|\mu\| \leq k\right\}<d \kappa,
$$

where $\kappa=\kappa(k)$ and $d$ is the $L_{1}$-distance between the differential $\psi_{0}$ and the line

$$
c \frac{\zeta_{0}-1}{(z-1)\left(z-\zeta_{0}\right)}, \quad c \in \mathbb{C} .
$$

In contrast to Theorems 3.1 and 4.3, the bound (4.15) is not sharp.

Similar results are valid also for the over-normalized functions in bounded quasidisks. We illustrate those again on the coefficient problem:

Find $\max \left|a_{n}\right|(n \geq 2)$ for the functions $f \in S_{k}(\infty)$ leaving a given set $e=\left(e_{1}, \ldots, e_{m}\right) \subset$ $\partial\left(\mathbb{D}^{*} \backslash\{\infty\}\right)$ fixed (hence some $\left.e_{s}=\infty\right)$.

Theorem 4.5. For any $n \geq 2$, there is a number $k_{n}(e)<1$ such that for $k \leq k_{n}$ and all $f \in S_{k}(\infty)$, which fix a given set $e=\left(e_{1}, \ldots, e_{m}\right)$, we have the sharp bound

$$
\max _{\|\mu\| \leq k}\left|a_{n}\left(f^{\mu}\right)\right|=\max _{|t|=\kappa}\left|a_{n}\left(f^{t\left|\psi_{n}\right| / \psi_{n}}\right)\right|=\frac{2 d_{n} \kappa}{n-1}+O\left(\kappa^{2}\right),
$$

where $\kappa(k)$ is again the bound for Teichmüller norms, and similar to (4.10),(4.11),

$$
\psi_{n}(z)=c z^{-n-1}+\sum_{1}^{m} \xi_{s} \rho_{s}(z), \quad d_{n}=\inf _{\mathcal{L}(e)}\left\|\psi_{n}-\psi\right\|_{1} .
$$


The constants $c, \xi_{s}$ are determined from the equations of type (4.12), and the remainder in (4.16) is estimated uniformly for all $k \leq k_{n}$.

Remark 2. The distortion bounds of type (4.9) given by Theorem 4.4 and its corollaries hold in somewhat weakened form (up to terms $O\left(k^{2}\right)$ ) for the maps preserving an infinite subset $e$ in $D$, provided that the corresponding class $\Sigma_{k}\left(D^{*}, 1, e\right)$ contains the functions $f^{\mu} \neq \mathrm{id}$.

The proof is similar but now the quadratic holomorphic differentials $\psi_{e}$ defining the extremal functions are represented instead of (4.10) in the form

$$
\psi_{e}=\psi_{0}+\psi, \quad \psi \in \mathcal{L}(e)
$$

and there are no variations of type Lemma 4.3 for the infinite sets.

4.6. Shift-like theorems. The above theorems also make a progress in solving old question related to Teichmüller's Verschiebungssatz of 1944. This theorem provides explicitly the extremal map among quasiconformal automorphisms of the disk $\mathbb{D}$ preserving fixed all boundary points and moving a given point $z_{0} \in \mathbb{D}$ into a given $w_{0}$. It found interesting applications. There were given some its modifications, but until now no finite dimensional analogs (i.e., for finite sets of fixed boundary points) have been established. Theorems 3.1 and 4.4 give a particular answer to this question.

Consider holomorphic functionals $J(f)$ of the form

$$
J(f)=J\left(f\left(z_{1}\right), \ldots, f\left(z_{n}\right)\right)
$$

depending on the values of maps $f \in \Sigma\left(D^{*}\right)$ in the distinguished points $z_{j} \in D \cup D^{*}$. Let again $J(\mathrm{id})=0, \operatorname{grad} J(\mathrm{id}) \neq 0$. For any fixed finite set $e=\left(e_{1}, \ldots, e_{m}\right) \subset \partial \mathbb{D}^{*}$, the maximal value of $|J(f)|$ on the class $\Sigma\left(\mathbb{D}^{*}, e\right)$ is obtained via (4.9) and growths monotonically with the dilatation $k$. Thus, one can prescribe $r<\max _{\Sigma\left(\mathbb{D}^{*}, e\right)}|J(f)|$ and ask on the minimal dilatation $k$ on which such $r$ is attained on the maps $f^{\mu} \in \Sigma\left(D^{*}\right)$ preserving the points of the set $e$. Theorem 4.4 yields the following geometric result.

Theorem 4.6. For small $r>0$, the minimal dilatation, on which any functional (4.17) attains its level surface $L_{r}=\{|J(f)|=r\}$, equals

$$
\kappa=r / d+O\left(r^{2}\right)
$$

with d defined by (4.11). This estimate is sharp.

The problem of establishing sharp explicit global distortion estimates for over-normalized maps, even such as Theorem 3.1, is very complicated and remains open.

I am thankful to the referee for his comments and suggestions.

\section{REFERENCES}

[1] L. Bers, Fiber space over Teichmüller spaces, Acta Math. 130 (1973), 89-126.

[2] P. Biluta and S.L. Krushkal, On the question of extremal quasiconformal mappings, Soviet Math. Dokl. 11 (1971), 76-79.

[3] S. Dineen, R.M. Timoney and J.P. Vigué, Pseudodistances invariantes sur les domains d'une espace localement convexe, Ann. Scuola. Norm. Sup. Pisa Cl. Sci.(4) 12 (1985), 515-529.

[4] C.J. Earle and J.J. Eells, On the differential geometry of Teichmüller spaces, J. Analyse Math. 19 (1967), 35-52.

[5] C.J. Earle and I. Kra, On sections of some holomorphic families of closed Riemann surfaces, Acta Math. 137 (1976), 49-79. 
[6] C.J. Earle, I. Kra and S. L. Krushkal, Holomorphic motions and Teichmüller spaces, Trans. Amer. Math. Soc. 343 (1994), 927-948.

[7] C.J. Earle and S. Mitra, Variation of moduli under holomorphic motions, In the tradition of Ahlfors and Bers (Stony Brook, NY, 1998), Contemp. Math. 256, Amer. Math. Soc., Providence, RI, 2000, pp. 39-67.

[8] F.P. Gardiner and N. Lakic, Quasiconformal Teichmüller Theory, Amer. Math. Soc., 2000.

[9] A.A. Golubev and S.Yu. Graf, Extremal problems on the classes of quasiconformal embeddings of Riemann surfaces, Siberian Adv. Math. 11 (2001), no. 4, 47-67.

[10] G.M. Goluzin, Geometric Theory of Functions of Complex Variables, Transl. of Math. Monographs, vol. 26, Amer. Math. Soc., Providence, RI, 1969.

[11] H. Grunsky, Koeffizientenbedingungen für schlicht abbildende meromorphe Funktionen, Math. Z. 45 (1939), 29-61.

[12] V.Ya. Gutlyanskii and V.I. Ryazanov, Geometric and Topological Theory of Functions and Mappings (Geometricheskaya $i$ topologicheskaya teoria funkcii $i$ otobrazhenii), Naukova Dumka, Kiev, 2011 (Russian).

[13] E. Hille and R.S. Phillips, Functional Analysis and Semigroups (Colloquium Publication Series 31), Amer. Math. Soc., Providence, R.I., 1957.

[14] I. Kra, The Carathéodory metric on abelian Teichmüller disks, J. Anal. Math. 40 (1981), 129-143.

[15] S.L. Krushkal, Quasiconformal Mappings and Riemann Surfaces, Wiley, New York, 1979.

[16] S.L. Krushkal, Grunsky coefficient inequalities, Carathéodory metric and extremal quasiconformal mappings, Comment. Math. Helv. 64 (1989), 650-660.

[17] S.L. Krushkal, Exact coefficient estimates for univalent functions with quasiconformal extension, Ann. Acad. Sci. Fenn. Ser. A.I. Math. 20 (1995), 349-357.

[18] S.L. Krushkal Plurisubharmonic features of the Teichmüller metric, Publications de l'Institut Mathématique-Beograd, Nouvelle série 75(89) (2004), 119-138.

[19] S.L. Krushkal, Extremal problems for Fredholm eigenvalues, Israel J. Math. 172 (2009), 279-307.

[20] S.L. Krushkal, Hyperbolic metrics on universal Teichmüller space and extremal problems, Ukrains'kii Matematychnii Visnyk 8 (2011), 557-579; J. of Math. Sciences 182, no. 1 (2012), $70-86$.

[21] S.L. Krushkal, Strengthened Grunsky and Milin inequalities, Contemp. Math. 667 (2016), 159-179.

[22] S.L. Krushkal, The Grunsky function and Carathéodory metric of Teichmüller spaces, Complex Variables and Elliptic Equations 61 (2016), 803-816.

[23] S. L. Kruschkal und R. Kühnau, Quasikonforme Abbildungen - neue Methoden und Anwendungen, Teubner-Texte zur Math., vol. 54, Leipzig, 1983.

[24] S.L. Krushkal and R. Kühnau, Quasiconformal reflection coefficient of level lines, Contemp. Math. 553 (2011), 155-172.

[25] R. Kühnau, Verzerrungssätze und Koeffizientenbedingungen vom Grunskyschen Typ für quasikonforme Abbildungen, Math. Nachr. 48 (1971), 77-105.

[26] R. Kühnau, Über die Werte des Doppelverhältnisses bei quasikonformer Abbildung, Math. Nachr. 95 (1980), 237-251.

[27] R. Kühnau, Wann sind die Grunskyschen Koeffizientenbedingungen hinreichend für Qquasikonforme Fortsetzbarkeit? Comment. Math. Helv. 61 (1986), 290-307.

[28] R. Kühnau und W. Niske, Abschätzung des dritten Koeffizienten bei den quasikonform fortsetzbaren schlichten Funktionen der Klasse S, Math. Nachr. 78 (1977), 185-192.

[29] D. Minda, The strong form of Ahlfors' lemma, Rocky Mountain J. Math. 17 (1987), 457-461.

[30] Chr. Pommerenke, Univalent Functions, Vandenhoeck \& Ruprecht, Göttingen, 1975. 
[31] E. Reich and K. Srebel, Extremal quasiconformal mappings with given boundary values, Contribution to Analysis (L.V. Ahlfors et al., eds.), Academic Press, New York, 1974, pp. 375-392.

[32] H. Renelt, Extremalprobleme bei quasikonformen Abbildungen unter höheren Normierungen, Math. Nachr. 66 (1975), 125-143.

[33] H.L. Royden, Automorphisms and isometries of Teichmüller space, Advances in the Theory of Riemann Surfaces (Ann. of Math. Stud., vol. 66), Princeton Univ. Press, Princeton, 1971, pp. 369-383.

[34] M. Schiffer and D. Spencer, Functionals of finite Riemann Surfaces, Princeton Univ. Press, Princeton, 1954.

[35] G. Schober, Univalent Functions - Selected Topics, Lecture Notes in Math. 478, Springer, Berlin, 1975.

[36] V.G. Sheretov, Classical and Quasiconformal Theory of Riemann Surfaces, Computer Reseacrh Institute "Regular and Chaotic Dynamics", Moscow- Izhevsk, 2007 (Russian).

[37] K. Strebel, On the existence of extremal Teichmueller mappings, J. Anal. Math. 30 (1976), 464-480.

[38] H. Tanigawa, Holomorphic families of geodesic discs in infinite dimensional Teichmüller spaces, Nagoya Math. J. 127 (1992), 117-128.

[39] O. Teichmüller, Ein Verschiebungssatz der quasikonformen Abbildung, Deutsche Math. 7 (1944), 336-343.

[40] E. Vesentini, Complex geodesics and holomorphic mappings, Sympos. Math. 26 (1982), 211230.

Department of Mathematics, Bar-Ilan University

5290002 Ramat-Gan, Israel

and Department of Mathematics, University of Virginia,

Charlottesville, VA 22904-4137, USA 